\title{
An Implicit Time-Stepping Scheme for Rigid Body
Dynamics with Coulomb Friction *
$\begin{array}{cc}\text { David Stewart } & \text { J.C. Trinkle } \\ \text { Dept. of Mathematics } & \text { Sandia National Labs } \\ \text { University of Iowa } & \text { Albuquerque, NM 87185-1008. }\end{array}$
}

Iowa City, IA 52242

Abstract. In this paper a new time-stepping method for simulating systems of rigid bodies is given. Unlike methods which take an instantaneous point of view, our method is based on impulse-momentum equations, and so does not need to explicitly resolve impulsive forces. On the other hand, our method is distinct from previous impulsive methods in that it does not require explicit collision checking and it can handle simultaneous impacts. Numerical results are given for one planar and one threedimensional example, which demonstrate the practicality of the method, and its convergence as the step size becomes small.

\section{Introduction}

Our world can be viewed as a huge multibody system composed of numerous multibody subsystems which interact through unilateral contacts. In fact, most planned changes to our environment could not be carried out if frictional contacts could not be made and broken at will. The act of retrieving a banana from a fruit bowl can be easily accomplished by a human who "understands" the physics of contact and friction. Building a bridge, transmission, or spacecraft requires humans with such knowledge.

Ultimately, roboticists would like to build robots with human-like understanding of contact and friction in order to (at least partially) automate tedious and/or dangerous manufacturing and clean-up tasks. However, to have any chance of succeeding in this endeavor, we must first fully understand the relevant phenomena ourselves and be able to accurately predict system behavior. This implies an ability to solve the equations of accurate dynamic models over time, given input functions of time. However, the roboticist's task goes on beyond the short-term goal of solving the forward problem just mentioned. To endow robots with a useable knowledge of multibody systems. one must solve the inverse, or planning, problem.

* This research was partially supported by NSF grant IRI-930-4734. THECB grant ARP-999903-078. Sandia Corporation under US DOE contract DE-AC04-94AL-85000.
Previous work that will eventually help us develop general algorithms for planning tasks with contact include studies on mathematical models of multibody systems, theories of solution existence and uniqueness for those models. methods for simulation. algorithms for motion planning for constrained systems, and implementations of planners based on these models (despite their restricted domains) $[3,6,9,12,13,18,22,24]$. We contend that one of the primary reasons general algorithms have not been developed is the difficulty in solving the general forward problem. Both rigid body and compliant body methods have been developed (e.g.. [2. 7, 11, 14, 15, 19]) and each has its shortcomings. For example. constraint-based rigid body simulators occassionally fail due to solution nonexistence [23]. They also require switching between impulse-momentum and force-acceleration models as contacts form and break. Cluttered environments with lots of bouncing can stall these methods. Impulse-based methods bog down when the physics of the situation suggests many static contacts, as would occur when simulating a house full of books, furniture, etc. Finite element methods increase the computational burden and also suffer from solution nonuniqueness when applied to contact problems [10]. With all of these methods, there is additional overhead of collision detection, which can become the bottleneck in cluttered environments with high-resolution geometric models. .

The above concerns have motivated the method presented here, which is best classified as an impulse-based method. The predominant influences on our approach are the works of Mirtich [15], Moreau [17], MonteiroMarques [16]. However, our method avoids several of the drawbacks of their methods. By formulating our timestepping method in terms of impulses, velocities, $A N D$ positions, we derive several benefits: (1) all contact forces, whether finite or instantaneously infinite are treated uniformly, as impulses accruing over-a single time step, (2) we avoid the need to explicitly determine the times of impacts and to switch to an impulse-momentum law at those 


\section{DISCLAIMER}

This report was prepared as an account of work sponsored by an agency of the United States Government. Neither the United States Government nor any agency thereof, nor any of their employees, make any warranty, express or implied, or assumes any legal liability or responsibility for the accuracy, completeness, or usefulness of any information, apparatus, product, or process disclosed, or represents that its use would not infringe privately owned rights. Reference herein to any specific commercial product, process, or service by trade name, trademark, manufacturer, or otherwise does not necessarily constitute or imply its endorsement, recommendation, or favoring by the United States Government or any agency thereof. The views and opinions of authors expressed herein do not necessarily state or reflect those of the United States Government or any agency thereof. 


\section{DISCLAIMER}

Portions of this document may be illegible in electronic image products. Images are produced from the best available original document. 
times, and (3) no special formulation is required to handle multiple "simultaneous" impacts, so that the simulation step size is fairly insensitive to the complexity of scene. An additional benefit that results from the particulars of our development, is that solutions always exist for the single contact case, and a minor modification discussed in \$3.3 guarantees solution existence for all multiple contact cases. To clarify the implications of the points just raised, our method can: (1) step rapidly through time periods in which many collision are occuring in rapid succession (e.g., a "break" shot in pocket billiards), (2) handle situations in which contact forces are instantaneously infinite without a collision (see [2] for an example).

\section{Dynamic Model}

Let $t$ denote time and $q(t), v(t) \in \Re^{n}$ denote the generalized coordinate and velocity vectors of a system of rigid bodies. ${ }^{1}$ The Newton-Euler equations of this system with multiple unilateral contacts can be written in the following well-known form:

$$
M I(q) \frac{d v}{d t}=k(q, v)+\rho,
$$

where $M$ is the positive definite, symmetric inertia matrix, $k$ is the vector of Coriolis, centripedal, other external generalized forces and smooth generalized contact forces such as those arising from viscous and Stribeck effects. and $\rho$ is the sum of the normal and dry friction components of the generalized contact forces. The dry friction forces can be constrained to lie within the usual Coulomb friction cone, but our method admits more general friction limit surfaces [5]. We only require that for a given normal load, the friction force (and moment for distributed contacts) is an element of a convex subset of the space of the other contact force components. As will be seen, our method naturaily enforces the maximum work inequality for sliding contacts. These characteristics are typical of many useful dry friction models $[8,25]$.

Due to the fact that the bodies have nonzero volume, some values of $q$ correspond to interpenetration. The (possibly disconnected) portion of configuration space (Cspace) corresponding to interpenetration will be denoted as $\bar{C}$. Its complement $\mathcal{C}$ is the admissible region, whose boundary $\partial \mathcal{C}$ is assumed to be piecewise smooth. Under the rigid assumption, $q(t)$ must remain in $\mathcal{C}$ for all time.

In what follows, we will cast the Newton-Euler equation (1), nonpenetration constraints, and a friction law as a linear complementarity probem in a discrete-time form suitable for use in simulation. As will be seen later, the complementarity form is intrinsic to the problem.

\footnotetext{
${ }^{1}$ For convenience, we have assumed a three-parameler representation of body orientation.
}

\section{Complementarity Formulation of the Time-Stepping Algorithm}

The standard linear complementarity problem (LCP) is stated as follows [4]. Determine vectors $w$ and $z$ such that the following conditions are satisfied:

$$
w \geq 0 \quad \begin{gathered}
w=R z+u \\
z \geq 0
\end{gathered} \quad w^{T} z=0
$$

where $R$ is a known square matrix, $u$ is a known vector of compatible length, and the superscript " $T$ " is the transpose operator. When the matrix $R$ satisfies certain properties, solutions are known to exist. Under slightly more restrictive assumptions. a solution is guaranteed to be computable by Lemke's algorithm; a pivoting method similar to the simplex algorithm for linear programming. When $w$ is a nonlinear function of $z$, the other constraints remaining the same, we have a nonlinear complementarity problem (NCP). It is generally difficult to prove solution existence for an $\mathrm{NCP}$, and one must often resort to general nonlinear programming algorithms to find solutions.

We begin the development of our method under the assumptions of isotropic Coulomb friction, only one contact. and a simple admissible space assumed to be represented by a single scalar inequality:

$$
\mathcal{C}=\{q \mid f(q) \geq 0\} \subset \Re^{n} .
$$

In order to capitalize on the efficient tools of LCP theory, we linearize the model: first. by replacing the friction cone with a polyhedral approximation, and second, by approximating the nonpenetration constraint $f(q) \geq 0$ by the half-space: $n^{T} q \geq \alpha_{0}$, where $n$ is the gradient of $f$ with respect to $q$. (this assumption will be relaxed in $\$ 3.4$ ). Note that if there is only one moving body, then, with the proper choice of generalized coordinates and scaling of $f(q), n$ is the six-simensional unit wrench of the normal component of the contact force.

Letting $c_{\mathrm{n}}$ denote the magnitude of the normal contact force and $c_{\xi}$ frictional component of the contact force, the circular friction cone can be approximated by a polyhedral cone $\mathcal{F}$ (see Figure 1):

$$
\mathcal{F}(q)=\left\{c_{\mathrm{n}} n+D \beta \mid c_{\mathrm{n}} \geq 0, \beta \geq 0, e^{T} \beta \leq \mu c_{\mathrm{n}}\right\},
$$

where $e=[1,1, \ldots, 1]^{T} \in \Re^{k}$ with $k$ being the number of edges of the polyhedral approximation, $\beta \in \Re^{k}$ is a vector of weights. The columns of $D$ are direction vectors that positively span the space of possible generalized friction forces. Thus if $s$ is a vector in that subspace, then $d_{j}^{T} s \geq 0$ for all $j$ implies $s=0$. Note that $n$ and $d_{j}$ are unit vectors for isotropic Coulomb friction modeled in the space of the contact force. However, the lengths of the 


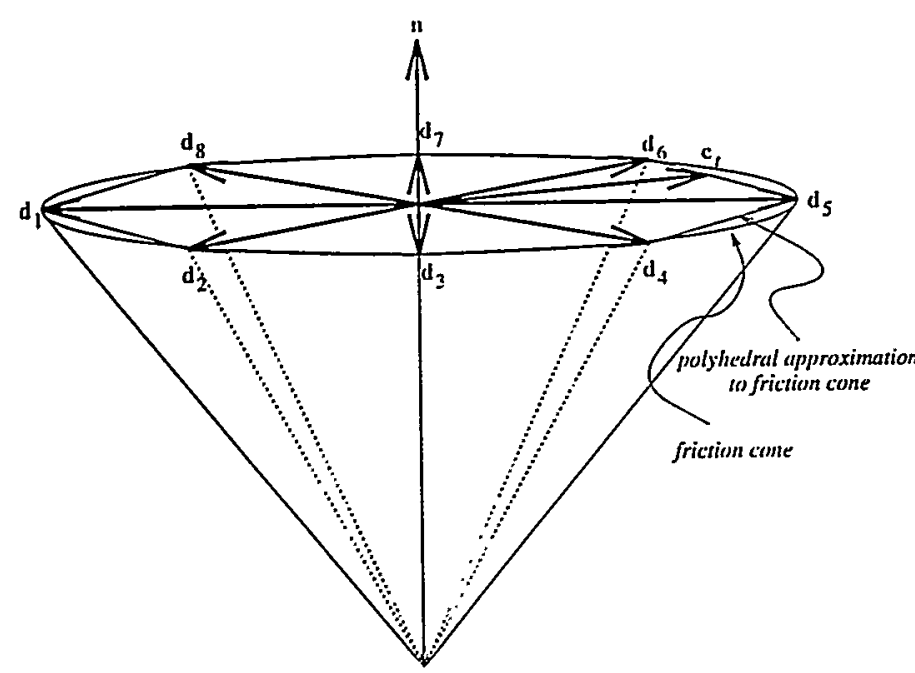

Figure 1: Circular friction cone and polyhedral approximation

$d_{j}$ would vary for anisotropic Coulomb friction. In addition, when these direction vectors are transformed into $\mathrm{C}$-space, they generally are no longer of unit length (e.g., for a single moving rigid body, the unit vectors map into unit wrenches of length 6 ).

We also need the following contact constraint. which is the source of complementarity in our model:

$$
\rho^{T} f(q)=0 .
$$

This constraint ensures that the contact force can only be nonzero when contact exists. Note that we have not yet defined a relationship to determine the direction of the contact force during sliding. This relationship will be incorporated during the discretization below of the model $(1-4)$.

\subsection{Model Discretization}

Let $h$ denote the step size and $t^{l}$ the current time, for which we have estimates the position $q^{l}=q\left(t^{l}\right)$ and velocity $v^{l}=v\left(t^{l}\right)$ of the system. Our goal is to derive approximations of the position $q^{l+1}$ and velocity $v^{l+1}$ that satisfy the model and the maximum work inequality ${ }^{2}$ at the end of the next time step. Approximating $\frac{d v}{d t}$ by $\frac{v^{l+1}-v^{l}}{h}, \frac{d q}{d t}$ similarly, and substituting the results into equation (1) yields the following discrete-time, NewtonEuler equations:

$$
\begin{aligned}
M \cdot\left(v^{l+1}-v^{l}\right) & =h n c_{\mathrm{n}}+h D \beta+h k \\
q^{l+1}-q^{l} & =h v^{l+1},
\end{aligned}
$$

\footnotetext{
${ }^{2}$ The maximum work incquality will set the direction of the friction force during sliding contact.
}

where $h c_{n}$ and $h D \beta$ are the impulses of the normal contact force and frictional contact force over the the time period from $t^{l}$ to $t^{l+1}$. Observe that these expressions appear to be linear in the unknowns $q^{l+1}, v^{l+1}, c_{\mathrm{n}}$, and $\beta$. However, $M, n, D$, and $k$ also depend on $q(t)$ and $v(t)$. Since our objective is to obtain a solution for all variables at the end of each time step, we would like to evaluate these quantities at $q^{l+1}$ and $v^{l+1}$, causing the discrete-time equations to become nonlinear. However, in order to preserve linearity, these quantities will be evaluated at $q=q^{l}+h v^{l}$ and $v=v^{l}$. Later, in $\S 3.4$ we will present a method for solving the nonlinear problem.

To complete the time-stepping equations, the discretetime Newton-Euler equation must be combined with the following system of inequalities. Their purpose is to produce the correct friction force and nonpenetration behavior with the aid of a new scalar unknown $\lambda$ (explained below):

$$
\begin{aligned}
n^{T} q^{l+1} \geq \alpha_{0}, & c_{\mathrm{n}} \geq 0, \\
\lambda e+D^{T} v^{l+1} \geq 0 . & \beta \geq 0, \\
\mu c_{\mathrm{n}}-e^{T} \beta \geq 0 . & \lambda \geq 0,
\end{aligned}
$$

with the complementarity conditions:

$$
\begin{aligned}
\left(n^{T} q^{l+1}-\alpha_{0}\right) c_{\mathrm{n}} & =0 . \\
\left(\lambda e+D^{T} v^{l+1}\right)^{T} 3 & =0 \\
\left(\mu c_{\mathrm{n}}-e^{T} 3\right) \lambda & =0 .
\end{aligned}
$$

- Separation: Separation is indicated by $n^{T} q^{l+1}>$ $\alpha_{0}$. In this case, equations (7) and (10) imply that $c_{\mathrm{n}}=0$. Substituting into the left inequality in (9) implies that $\beta=0$, thereby satisfying equations $(8.9 .11,12)$. These conclusions are independent of $v^{l+1}$ since there is no contact. Also, $\lambda$ may take on any positive value satisfying the left inequality in (8) and has no meaningful physical interpretation.

- Sliding: For sliding, $v^{l-1}$ is of nonzero length and in the span of the columns of $D$. Since the vectors $d_{j}$ positively span the space containing $v^{l+1}$, the vector $D^{T} v^{l+1}$ contains at least one strictly negative element. In order to satisfy the left inequality in (8), $\lambda$ must be strictly positive. Moreover, we have, $\lambda \geq \lambda^{*}=\max _{i}\left\{-d_{i}^{T}\right\} v^{l+1}>0$. (If $\lambda>\lambda^{*}$, then equation (12) implies that the left inequality in (9) must be strictly satisfied (i.e., $\mu c_{n}=e^{T} \beta$ ). ) Therefore, the sum of the elements of $\beta$ must be $\mu c_{n}$. Equation (11) with inequality (8) implies that typically only one element of $\beta$ will be nonzero, and this one will be the one which maximizes power dissipation. In nongeneric cases where more than one element of $\beta$ is nonzero, those elements must still sum to $\mu c_{n}$, which constrains the friction force to the facet of the 
linearized friction cone formed by the corresponding $d_{j}$ vectors. $^{3}$

- Rolling: In this case, $v^{t+1}=0$, so the left inequality in (8) is equivalent to $\lambda \geq 0$.

Case 1: Choosing $\lambda=0$ reduces our system of inequalities to the left one in (9) and $\beta \geq 0$. This inequality system simply allows the contact impulse to range over the interior and surface of the linearized friction cone.

Case 2: Choosing $\lambda>0$ implies that $\beta=0$ and $\mu c_{\mathrm{n}}=0$. This is a nongeneric sort of rolling that occurs by chance in the absence of a frictional impulse.

Several aspects of the discrete-time dynamic equations (5-12) should also be noted.

1. It is a mixed linear complementarity system as described in Cottle, Pang and Stone[4, pp. 29-30]. By solving for $v^{l+1}$ and $q^{l+1}$ in terms of the other quantities, a "pure" LCP can be obtained.

2. The average normal contact force $c_{n}$, and therefore $\beta$. can only be nonzero when there is contact at at the end of the interval,(i.e., $n^{T} q^{l+1}=\alpha_{0}$ ). Thus there is no need to explicitly "turn on" and "turn off" the contact forces in this method.

3. It was shown in [21] that solutions to this LCP always exist and can be found by Lemke's algorithm.

\subsection{Multiple contacts}

Multiple contacts can be easily incorporated into this framework. The additional data needed to form the LCP for a problem with $N$ contacts for a given time step follow. The vector $n^{(j)}$, is the normal vector for the $j^{t / 2}$ contact, transformed to generalized coordinates: and $\alpha_{0}^{(j)}$ the corresponding scalar for locating the boundary of the $j^{\text {th }}$ half-plane. The matrix $D^{(j)}$. is formed from the vectors spanning the friction force space at the $j^{\text {th }}$ contact. transformed to generalized coordinates. The scalar $\mu^{(j)} \geq 0$, is the coefficient of friction for the $j^{\text {th }}$ contact.

There are also new variables for each contact: $c_{n}^{(J)}$ (the average normal force for the $j^{\text {th }}$ contact over the time step), $\beta^{(j)}$ (the coefficients for the frictional impulse for the $j^{\text {th }}$ contact), and $\lambda^{(j)}$. With these data and variables. the formulation of the time-stepping method for $N$ contacts is given by:

$$
M \cdot\left(v^{l+1}-v^{l}\right)=\sum_{j=1}^{N}\left(n^{(j)} c_{i 1}^{(j)}+D^{(j)} \beta^{(j)}\right)+h k(13)
$$

\footnotetext{
${ }^{3}$ As long as the set of friction forces defined by the vectors $d_{3}$ is convex, then the elements of $\beta$ that are nonzero comespond to a vertex. edge, or facet on that surface.
}

$$
q^{l+1}-q^{l}=h v^{l+1},
$$

with nonpenetration and friction cone constraints for $j=$ $1, \ldots, N$ contacts:

$$
\begin{array}{rlrl}
n^{(j)^{T}} q^{l+1} & \geq \alpha_{0}^{(j)}, & c_{\mathrm{n}}^{(j)} \geq 0 \\
\lambda^{(j)} e^{(j)}+D^{(j) T} v^{l+1} & \geq 0, & \beta^{(j)} \geq 0 \\
\mu^{(j)} c_{\mathrm{n}}^{(j)}-e^{(j) T} \beta^{(j)} & \geq 0, & & \lambda^{(j)} \geq 0
\end{array}
$$

with the complementarity conditions for $j=1, \ldots, N$ :

$$
\begin{aligned}
{\left[n^{(j) T} q^{l+1}-\alpha_{0}(j)\right] c_{\mathrm{n}}^{(j)} } & =0, \\
{\left[\lambda^{(j)} e^{(j)}+D^{(j) T} v^{l+1}\right]^{T} \beta^{(j)} } & =0, \\
{\left[\mu^{(j)} c_{\mathrm{n}}^{(j)}-e^{(j) T} \beta^{(j)}\right] \lambda^{(j)} } & =0, .
\end{aligned}
$$

where $e^{(j)}$ is the column vector of ones of the appropriate length. It was shown in [21] that solutions to the multiple contact LCP exist and that Lemke's algorithm (modified to avoid cycling) will find a solution if the following conditions are satisfied. The normal vectors $n^{(j)}, j=1, \ldots, N$ must be linearly independent, and the only vector in both the span of the $n^{(j)}$ and the columns of $D^{(j)}, j=1, \ldots, N$ is the zero vector.

\subsection{Guaranteeing Solution Existence}

It has been found by Anitescu and Potra [1] that a simple modification to the above formulation guarantees solution existence and that Lemke's algorithm will find one. The modification is simply to replace the linearized nonpenetration constraint ( the left inequality in equation (7)) with the following tangential velocity constraint:

$$
n^{T} v^{l \div 1} \geq 0
$$

The primary drawback of using this inequality in place of the original one is that penetration can accrue as the configuration of the system moves along a concave portion of the boundary $f(q)$ in configuration space. This can be handled by simple projection methods, but care must be taken to avoid losing energy in the process.

\subsection{A Nonlinear Version}

One problem with the method as it is given, is that for real problems the admissible regions are not half-spaces, but are more general sets, usually with smooth boundaries. Thus the vector $n$, and $\alpha_{0}$, and the matrices $D$, depend crucially on the geometry of the problem, and vary from point to point. One particular consequence of this is the failure of the methods $(5-12)$ and $(13-20)$. This can happen where, due to the variation in $n(q)$ and $\alpha_{0}(q)$, while 
$q^{l+1}$ may be admissible for the linearization based at $q^{l}$, this does not mean that it would be admissible for the linearization at $q^{l+1}$. Because admissibility for $q^{l+2}$ for the linearization at $q^{l+1}$ is required, sufficient impulse to achieve this is applied at the step computing $q^{l+2}$. This reaction impulse for such a step can be far in excess of that for the real system, and has been observed to cause unphysical loss of contact.

To circumvent this problem, a nonlinear method should be employed. In particular. the complementarity condition:

$n^{T} q^{l+1}-\alpha_{0} \geq 0, \quad c_{\mathrm{n}} \geq 0, \quad\left(n^{T} q^{l+1}-a_{0}\right) c_{\mathrm{n}}=0$

should be replaced by the following nonlinear complementarity condition:

$$
f\left(q^{l+1}\right) \geq 0, \quad c_{\mathrm{n}} \geq 0, \quad f\left(q^{l+1}\right) c_{\mathrm{n}}=0,
$$

and $n, D, M$, and $k$ in equations (5) should be evaluated at $t^{l+1}$.

Fortunately, a fixed point iteration scheme can be used effectively to solve these nonlinear complementarity problems, as follows. At the first stage, an estimate for $\widehat{q}^{l+1}$ is computed (such as $\widehat{q}^{l+1}=q^{l}+h v^{l}$ ). Then LCP $(13-20)$ is solved using $D=D\left(\hat{q}^{l-1}\right)$ and $n=n\left(\hat{q}^{l+1}\right)$. The resulting estimate for $q^{l+1}$ is used as $\hat{q}^{l+1}$ for the next iteration. Provided the LCP has unique solutions and the solution operator is locally Lipschitz, then for sufficiently small $h$, the method is convergent. In practice the iteration converges very quickly.

\section{Numerical implementation and results}

The numerical method has been developed based on an implementation of Lemke's algorithm for solving LCP's. The version of Lemke's algorithm used is based on an explicit tableau, together with lexicographic degeneracy resolution. Rather than explicitly include all potentially active contact constraints. $f_{i}(q) \leq 0$, with $i$ ranging over all possible pairs of interpenetrating geometric features. only those that were active at the previous step, plus those violated at $q^{l}+h v^{l}$, are initially assumed to be active. Once the solution of the LCP has been found, the constraints are checked at the new value $q^{l+1}$ for fensibility. If any other constraints are found to be violated, they are added to the set of potentially active constraints. All of the methods have been implemented in $C$ using the Meschach library[20] to provide the linear algebra and basic data structures.

\subsection{Example 1: Falling Rod}

Consider a rod spinning in planar motion while falling onto a horizontal tabletop. The rod in Figure 2 is modeled as a two-dimensional object with straight parallel sides and semi-circular ends. The generalized co-ordinate vector for the rod is $q=[x, y, \theta]^{T}$, where $(x, y)$ are the co-ordinates of the center of mass ( $y$ vertical), and $\theta$ is the angle of the rod relative to the tabletop. The other physical properties of the rod are as follows: length (excluding the ends) $l=$ $0.5 \mathrm{~m}$; mass $m=1 \mathrm{~kg}$; half-width of rod (which is also the radius of the ends) $r=0.05 \mathrm{~m}$; moment of inertia $J=$ $0.002 \mathrm{~kg}-\mathrm{m}^{2}$; coefficient of friction between the rod and the table is $\mu=0.6$.

Using the above data. the mass matrix $M(q)$ is $\operatorname{diag}(m, m, J)$. The two constraint functions (one for each end) are $f_{\dot{ \pm}}(q)=y \pm(l / 2) \sin \theta \geq r$. The corresponding normal vectors are $n_{ \pm}(q)=[0,1, \pm(l / 2) \cos \theta]^{T}$, and the four friction vectors are $d_{i}=[ \pm 1,0, \pm(l / 2) \sin \theta]^{T}$.

The initial angle of the rod is $30^{\circ}$ to the horizontal, with zero initial translational velocity, but with an initial rotational velocity of $4 \mathrm{rad} \mathrm{s}^{-1}$. Gravitation is the only external force applied to the rod. With these initial conditions and physical properties. the state of the system after the first impact is such that acceleration-based time-stepping methods would fail due to solution nonexistence. However, our method has no difficulty with this situation (see Figure 2).

The simulated motion of the rod agrees with physical intuition (assuming zero coefficient of restitution). The rod begins by spinning and falling, and then collides with the table at time $t \approx 0.383$. After the impact, the tip of the rod slides right, stops, and begins sliding left before "slap-down." After full contact at time $t \approx 0.548$, the rod then remains horizontal and slides left for a short time ( 0.02 seconds). The rod is then at rest for the remainder of the integration. Note that fairly fine step sizes need to be used to properly resolve all the details of the behavior. Step sizes near $1 / 25$ th of a second are not sufficient.

To demonstrate convergence of the algorithm, graphs of the numerical results for different values of step size $h$ are shown in Figures 3, 4 and 5. Note the absence of numerical chattering in the solutions. However, there is a spike in the angular velocity for the first contact. This is because of the position-based time-stepping used: after the first step in which contact is made, $f(q)=0$ : on the following step, the velocity is made tangential to the contact surface. with $v^{T} \nabla f(q)=0$.

\subsection{Example 2: Colliding Spheres}

Next consider a set of three slightly separated balls in a line on the table. and another ball in the air thrown towards three balls. The ball in the air first hits the table at $t \approx 0.42$ seconds, and begins to roll. It then collides obliquely with the first ball in the line at $t \approx 0.59$ seconds (see Figures 7 and 8). 


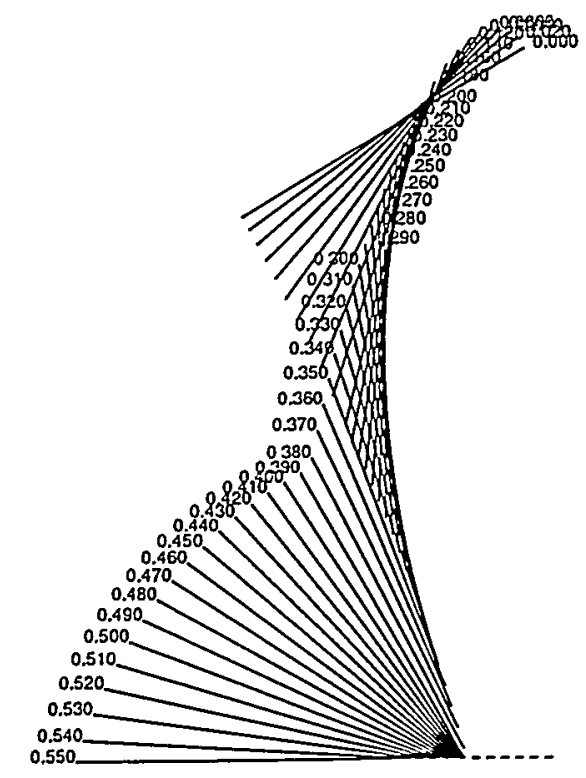

Figure 2: Falling and spinning rod, $h=0.0025$ (simulated time in seconds appears at one end of the rod).

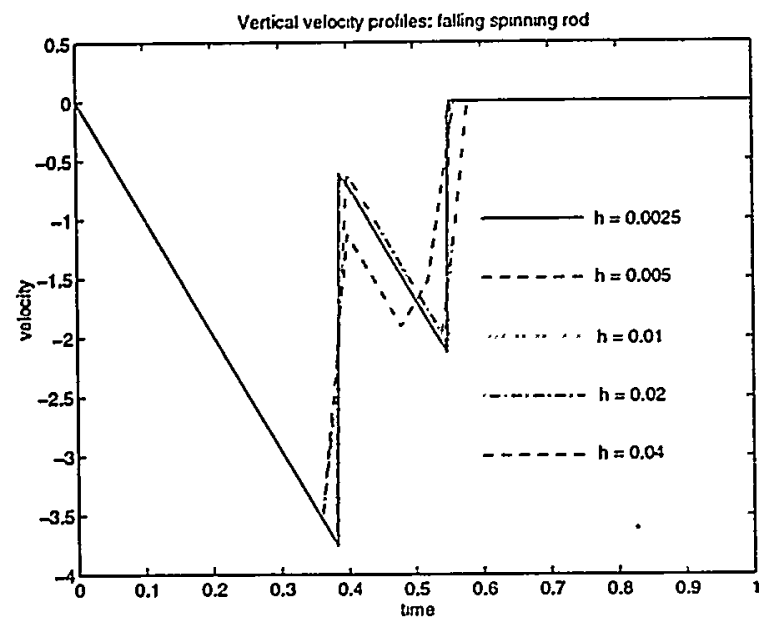

Figure 3: Vertical velocity of rod for various $h$ values

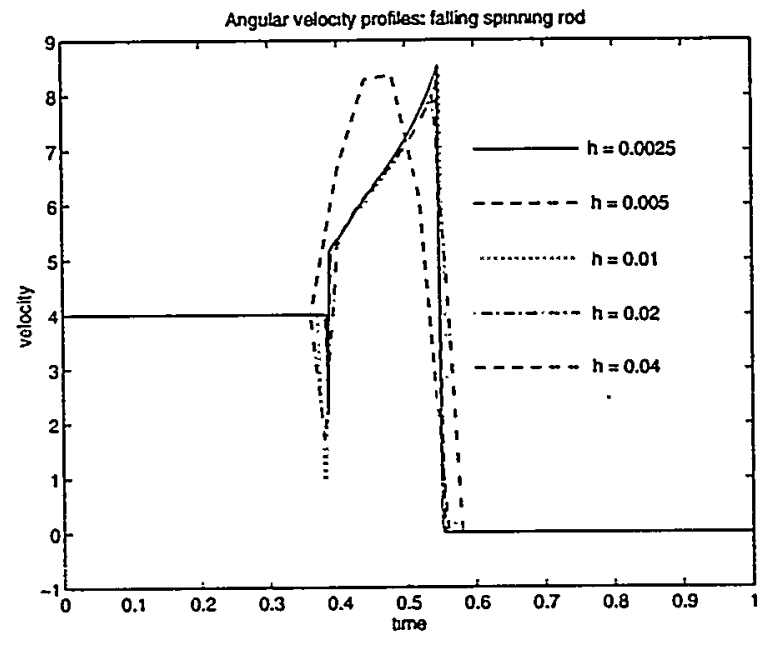

Figure 4: Angular velocity of rod for various $h$ values

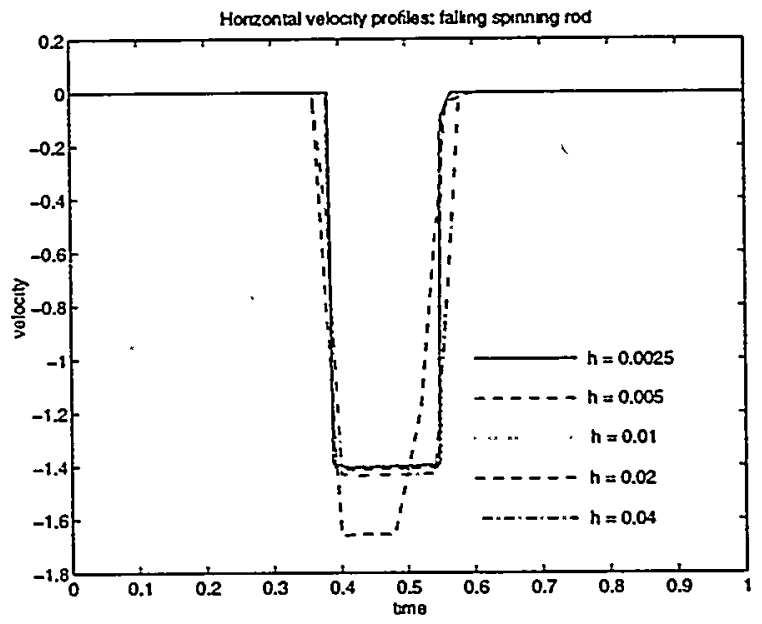

Figure 5: Horizontal velocity of rod for various $h$ values 


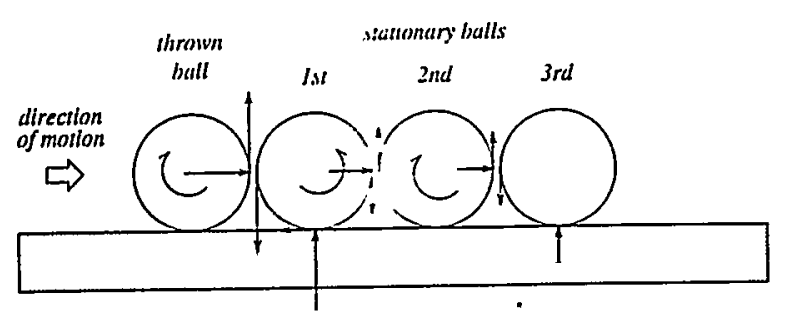

Figure 6: Impulses at collision of balls

The specific data for this problem is as follows: all balls are $1 \mathrm{~kg}$ in mass, have a radius of $0.1 \mathrm{~m}$, with moments of inertia $4 \times 10^{-3}=(2 / 5) \mathrm{mr}^{2}$; the coefficients of friction for all contacts is 0.4 . The initial positions of the balls (given as $x, y, z$ triples, with $z$ being the upward normal of the tabletop) are $[0,0,1.0] .11 .0,0.1],\left[1.2+10^{-5} ; 0,0.1\right]$ and $\left[1.4+2 \times 10^{-5}, 0,0.1 ;\right.$, and the initial velocities are $[1.5,0.1,0]$ for the first ball and zero for the others. Note the gaps of $10^{-5}$ between the stationary balls.

Eight friction direction vectors are used to approximate isotropic Coulomb friction at each contact. All vectors $d_{i}$ have unit length and are equally spaced around a circle tangential to the contact normal.

The gaps between the balls was chosen to ensure that multiple collisions occurred in the time-step of the initial collision, and to show that the cascade of frictional impulses transmitted through the line of balls matches ones intuition (see Figure 6). A frictional impulse applied at the first impact point lifts the thrown ball a small distance (not shown), due to the frictional impulse applied to it by the first stationary ball (indicated by a vertical arrow on the right side of the thrown ball). The corresponding downward impulse on the first stationary ball induces a vertical reaction impulse by the tabletop to prevent interpenetration. The frictional impulse from the thrown ball generates back-spin in the first stationary bail, and thus the table applies a frictional impulse resisting that rotation. Still during the same time step. contact is made with the second stationary ball, which applies an additional frictional impulse against the rotation of the first stationary ball. etc. Physically reasonable impulses propogate through to the end of the line of ball, all in the same time step. Note that the horizontal momentum of the thrown ball ultimately causes all the balls to move roughly to the right. but in different directions due to the oblique collision. The trajectories of the balls are shown in plan and elevation views in Figures 7 and 8, respectively.

Table 1 gives error estimates and variation estimates for the numerically generated solutions for step sizes $h=$ 0.0025 to $h=0.02$ using the solution for $h=0.00125$ as "exact". It also shows the variations of the numerically computed velocities, $\mathrm{V}\left(v^{h}\right)$. for different values of $h$. The
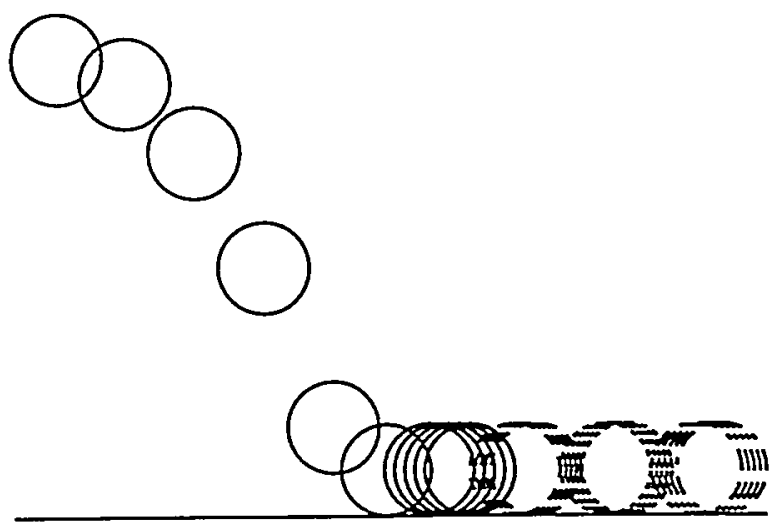

Figure 7: Elevation view of balls

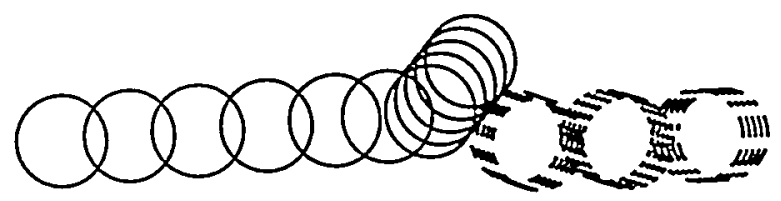

Figure 8: Plan view of balls

error measures used are $\int_{0}^{1}\left\|v^{h}(t)-v(t)\right\| d t$ for the velocities. and the supremum norm for the position errors. The $\infty$-norm is the norm used on $\Re^{n}$ (here $n=24$ ). The size of the LCP's solved for this problem were sometimes as large as $70 \times 70$ for 7 contacts. This is because there are eight direction vectors for each frictional forces, one variable for each normal force. and a $\lambda$ variable for each contact. It should be noted that the largest errors in the velocities were in the angular velocity components rather then the translation velocities: the angular velocity errors were about an order of magnitude larger. Table 1 shows that the errors are roughly of order $h$ for small values of $h$.

\section{Summary}

The impulse-based simulation method presented here for systems of rigid bodies with friction has several unique features. Unlike force-based formulations, the method al-

\begin{tabular}{|l|c|c|c|}
\hline Step size $h$ & Vel. error & Pos. error & Vel. variation \\
\hline 0.02 & 0.5050 & 0.2505 & 19.4046 \\
0.01 & 0.3523 & 0.2015 & 19.1728 \\
0.005 & 0.1657 & 0.0838 & 19.1702 \\
0.0025 & 0.0700 & 0.0298 & 19.0862 \\
0.00125 & - & - & 19.0690 \\
\hline
\end{tabular}

Table 1: Errors and variations for numerical solutions of four balls problems 
ways has a solution computable by Lemke's algorithm. Further, it is unnecessary to resolve the specific times of impact. Rather, one only needs to maintain a set of active constraints - those in danger of being violated by penetration.

As given in this paper, the coefficient of restitution is effectively zero. However. if one cares to explicitly determine collision times, one could use the velocities at those times and a Newton-type restitution hypothesis to resolve the collision. If one prefers Poisson's hypothesis, one can solve an additional complementarity problem for each collision formulated in [1], to obtain the post-collision system state.

\section{References}

[1] M. Anitescu and F.R. Potra. Formulating dynamic multirigid-body contact problems with friction as solvable linear complementarity problems. ASME Nonlinear Dyutunics. 14:231-247, 1997.

[2] D. Baraff. Issues in computing contact forces for nonpenetrating rigid bodies. Algorithmica. pages 292-352. October 1993.

[3] M. Cherif and K.K. Gupta. Planning for in-hand dexterous manipulation. In Procecedings, Third Workshop on $\mathrm{Al}$ gorithmic Foundations of Robotics, pages 103-117. A.K. Peters, Boston. MA. 1998.

[4] R. W. Coule. J.S. Pang, and R. E. Stone. The Linear Complementarily Problem. Academic Press, 1992.

[5] S. Goyal. Planar Sliding of a Rigid Body with Dry Friction: Limit Surfaces and Dynamics of Motion. PhD thesis, Cornell University Department of Mechanical Engineering, January 1989.

[6] L. Han, J.C. Trinkle. and Z.X. Li. The instantaneous kinematics and planning of Jextrous manipulation. In Proc. of 1997 IEEE Intrinational Sysmposium on Assembly and Task Planning (ISATP'97). pages 60-65, 1997.

[7] E. J. Haug, S. C. Wu. and S. M. Yang. Dynamics of mechanical systems with coulomb friction, stiction. impact and constraint addition, deletion $\mathrm{i}$, ii and iii. Mechanism and Machine Theory. 21:401-425, 1986.

[8] R.D. Howe and M.R. Cutkosky. Practical force-motion models for sliding manipulation. International Journal of Robotics Research, 1996. in press.

[9] L.E. Kavraki, F. Lamiroux, and C. Holleman. Towards planning for elastic objects. In Proceedings, Thirt Workshop on Algorit/mic Foundations of Robotics. piages 313326. A.K. Peters. Boston. .IA, 1998.

[10] A. Klarbring. Examples of non-uniqueness and nonexistence of solutions to quasistatic contact problems with friction. Ingenieur-Archiv. 56:529-541, 1990.

[11] C. Laugier. Models and rigid and deformable bodics. In Proceedings, Thiral Workshop on Algoritlmic Foundations of Robotics, pages 327-338. A.K. Peters, Boston, MA, 1998.

[12] S.M. Lavalle and J.J. Kuffner. Randomized kinodynamic motion planning. In Proceedings, IEEE International Conference on Robotics and Automation, pages 473-479, May 1999.

[13] K. Lynch. Nonprehensile Manipulation: Mechanics and Planning. PhD thesis, Carnegie Mellon University School of Computer Science, March 1996.

[14] D.W. Marhefka and D.E. Orin. Simulation nonlinear damping model. In Proceedings. IEEE International Conference on Robotics and Automation, pages 1662-1668, April 1996.

[15] B. Mirtich. Impulse-Based Dynamic Simulation of Rigid Body Systems. PhD thesis. University of California, at Berkeley. Department of Electrical Engineering and Computer Science, 1996.

[16] M. D. P. Monteiro-Marques. Differential inclusions in nonsmooth mechanical problems: Shocks and dry friction. Progress in Nonlinear Differential Equations and Their Applications, 9. 1993.

[17] J. J. Morcau. Unilateral contact and dry friction in finite ireedom dynamics. In Vonsmooth Mechanics and Applications, pages 1-82. Spnnger-Verlag, 1988. International Centre for Mechanical Sciences. Courses and Lectures $\# 302$.

[18] M. A. Pestkin and A. C. Sanderson. The motion of a pushed. sliding workpiece. IEEE Journal of Robotics and Attomation. 4(6):569-598. December 1988.

[19] F. Pfeiffer and Ch. Glocker. Wultibody Dynamics with Unilateral Contacts. Wiley, 1996.

[20] D.E. Stewart and Z. Leyk. Meschach: Matrix Computations in C. Australian National University, 1994. Proc, of the C.MA. $\# 32$.

[21] D.E. Stcwart and J.C. Trinkle. An implicit time-stepping scheme for rigid body dynamics with inelastic collisions and coulomb triction. International Journal of Numerical Methods in Engineering. 39:2673-2691. 1996.

[22] J.C. Trinkle and J. J. Hunter. A framework for planning dextcrous manipulation. In Proceedings, IEEE International Conference on Robotics and Automation, pages 1245-1251. April 1991.

[23] J.C. Trinkle. J.S. Pang, S. Sudarsky, and G. Lo. On dynamic multi-rigid-body contact problems with coulomb friction. Zeitschrift für Angewandte Mathematik und Mechanik, 77(4):267-279, 1997.

[24] Y.-T. Wang, V. Kumar, and J. Abel. Dynamics of rigid bodies undergoing multiple frictional contacts. In Proceedings, IEEE International Conference on Robotics and Automation. pages 2764-2769, May 1992.

[25] N. Xydass and I. Kao. Modeling of contact mechanics and friction limit surfaces for soft fingers in robotics with experimental results. International Joumal of Robotics Research. 18(9):941-951. 1999. 